\title{
INSPECCIÓN DE PUENTES DE HORMIGÓN ARMADO CON TERMOGRAFÍA INFRARROJA: ESTUDIO DE CASO
}

\author{
J. H. A. ROCHA, G. GALINDO-AÑEZ \\ Departamento de Ingeniería Civil, Facultad de Tecnología, Universidad Privada del Valle, Cochabamba, Bolivia
}

\section{RESUMEN}

El presente estudio tiene por objetivo verificar la aplicabilidad de la termografía infrarroja en la inspección de puentes a través de un estudio de caso en una localidad de Bolivia. La metodología consistió en el análisis de un puente de hormigón armado, ubicado en la localidad de Punata, Cochabamba - Bolivia. La inspección se realizó tanto en la superestructura como la infraestructura utilizando la cámara termográfica FLIR CAT S60. Los resultados obtenidos permiten evidenciar la existencia de anomalías en la losa de la superestructura, principalmente, donde se desarrollan diferencias de temperatura marcadas. También se observa que los resultados son diferentes de acuerdo con el sector del puente inspeccionado, la superestructura presenta mejores resultados con relación a la detección de defectos, lo cual se debe a la exposición directa al sol. Si bien la termografía infrarroja puede localizar las áreas con anomalías, no puede dar la naturaleza y el grado del daño.

Palabras clave: Termografía infrarroja; inspección; puentes.

\begin{abstract}
The present study aims to verify the applicability of infrared thermography in bridge inspection through a case study in a locality of Bolivia. The methodology consisted in the analysis of a reinforced concrete bridge, located in the town of Punata, Cochabamba - Bolivia. The inspection was carried out both in the superstructure and the infrastructure using the FLIR CAT S60 thermal imaging camera. The obtained results allow to demonstrate the existence of anomalies in the bridge deck, mainly, where marked temperature differences are developed. It is also observed that the results are different according to the sector of the inspected bridge, the superstructure has better results in relation to the detection of defects, which is due to direct exposure to the sun. While infrared thermography can locate areas with anomalies, it cannot give the nature and degree of damage.
\end{abstract}

Keywords: Infrared thermography; inspection; bridges.

\section{RESUMO}

O presente estudo tem como objetivo verificar a aplicabilidade da termografia infravermelha na inspeção de pontes através de um estudo de caso em uma localidade da Bolívia. A metodologia consistiu na análise de uma ponte de concreto armado, localizada na cidade de Punata, Cochabamba Bolívia. A inspeção foi realizada tanto na superestrutura quanto na infraestrutura, utilizando a câmera termográfica FLIR CAT S60. Os resultados obtidos permitem demonstrar a existência de anomalias na laje da superestrutura, principalmente, onde diferenças de temperatura acentuadas são desenvolvidas. Observa-se também que os resultados são diferentes de acordo com o setor da ponte inspecionada, a superestrutura apresenta melhores resultados em relação à detecção de defeitos, o que se deve à exposição direta ao sol. Enquanto a termografia infravermelha pode localizar áreas com anomalias, o ensaio não pode dar a natureza e o grau de dano.

Palabras-chave: Termografia infravermelha; inspeção; pontes. 


\section{INTRODUCCIÓN}

Los puentes se caracterizan por ser estructuras importantes para el desarrollo económico y relacionamiento entre comunidades; no obstante, estas estructuras son propensas a desarrollar diversos problemas (Mascia and Sartorti, 2011). En Bolivia, un estudio realizado en el tramo Cochabamba Santa Cruz, reveló que, de 35 puentes, 20 tienen severas observaciones (El Deber, 2015) y se reporta constantemente el colapso de estas estructuras, la mayoría localizadas en la región de Cochabamba (La Opinión, 2015), demostrando la ineficiencia de las entidades encargadas y la falta de programas periódicos de inspección y mantenimiento.

Para preservar la vida útil, garantizar la seguridad y funcionalidad de las diversas estructuras de hormigón armado, las actividades de inspección y mantenimiento deben ser consideradas desde el momento de su construcción (Bagavathiappan et al., 2013). Especialmente en los puentes, ya que estas estructuras se emplazan normalmente en ambientes agresivos y están expuestas directamente al tráfico, lo que lleva a que se deterioren de forma rápida, generando manifestaciones patológicas (Gucunski et al., 2015). La corrosión en el acero, fisuras, desagregación de hormigón, hongos y eflorescencias son las principales manifestaciones patológicas encontradas en puentes (Mendes et al., 2010; Pintan et al., 2015)

En la actualidad, la metodología más empleada para la identificación de problemas en puentes es la inspección visual, la cual se limita a identificar discontinuidades y manifestaciones patológicas superficiales, sin dar información de la gravedad del daño (Oh et al., 2013). Esto evidencia la falta de aplicación de distintas tecnologías para el diagnóstico de estructuras; en este sentido, en los últimos años se han estado utilizado diferentes ensayos no destructivos, los cuales pueden llegar a ser eficientes de acuerdo con el problema analizado, además de proveer información de la integridad estructural si es que los resultados se complementan (Rehman et al., 2016).

Entre los varios ensayos no destructivos para la inspección de puentes relatados en la literatura, surge la termografía infrarroja como una opción conveniente, pues presenta varias ventajas para la inspección: no precisa el contacto directo con la estructura y analiza áreas, a diferencia de la mayoría de los ensayos no destructivos que su rango está limitado a puntos (Rehman et al., 2016). Diversos estudios (Hiasa et al., 2016; Watase et al., 2015; Sultan and Washer, 2017; Vaguefi et al., 2015) demuestran su eficiencia en la detección de defectos internos tanto experimentalmente como en estructuras reales, pero también se relata que el ensayo es fuertemente afectado por las condiciones ambientales y que los resultados varían de acuerdo con el lugar de aplicación.

En este sentido, el presente artículo tiene por objetivo verificar la aplicabilidad de la termografía infrarroja en la inspección de puentes en la región de Punata, Cochabamba - Bolivia, la cual presenta características diferentes a los casos ya presentados en la literatura.

\section{METODOLOGÍA}

Para verificar la aplicabilidad de la termografía infrarroja en la inspección de puentes se realizó un estudio de caso. Se seleccionó un puente de hormigón armado localizado en la región de Punata, Cochabamba - Bolivia. Este sector se caracteriza por tener un clima de estepa con pocas precipitaciones durante el año y temperatura anual de $16.6^{\circ} \mathrm{C}$ (Climate, 2019). La Figura 1 presenta la localización del puente inspeccionado. 


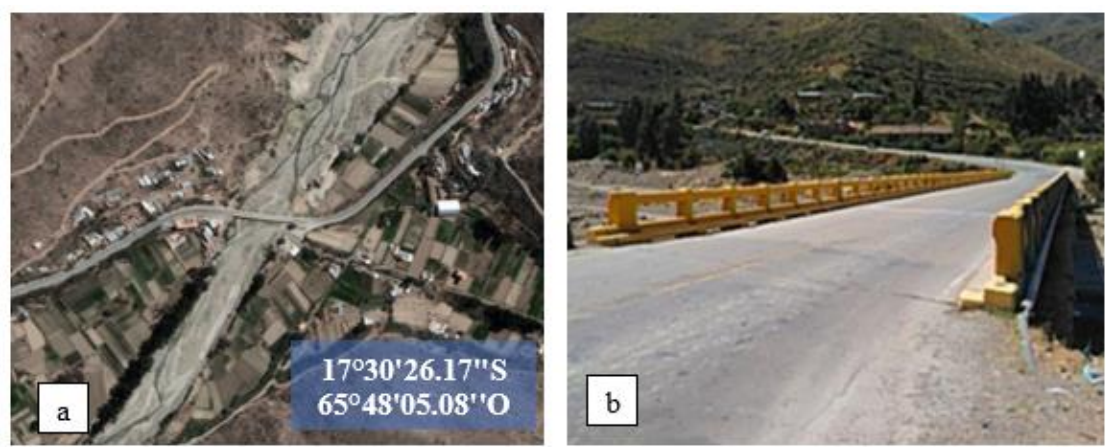

Figura 1. Puente estudiado: a) Localización y b) vista general

Si bien existen varias recomendaciones en los horarios para la inspección de puentes y la detección de defectos internos en concreto con termografía infrarroja, la mayoría de las investigaciones concuerdan que los horarios más adecuados son los próximos a medio día (Gucunski et al., 2013; Vaguefi et al., 2015; Watase et al., 2015; Rocha and Póvoas, 2019) o como indica la norma D4788-03 que debe existir un calentamiento del puente de por lo menos 3 horas (ASTM, 2013). Siguiendo estas recomendaciones, el horario de inspección adoptado para el presente estudio fue entre las 10:00 y 12:00. La temperatura ambiente registrada durante la inspección fue de $21^{\circ} \mathrm{C}$ y la humedad relativa de $48 \%$.

Se utilizó la cámara termográfica FLIR CAT S60, cuyas características se encuentran en la Tabla 1. La cámara termográfica fue posicionada entre 1 y 3 metros de los elementos inspeccionados de forma de obtener una buena resolución en los termogramas. La forma de aplicación fue pasiva, solo requiriendo el sol como fuente de calentamiento en la estructura.

Tabla 1. Características de la cámara termográfica

\begin{tabular}{|c|c|}
\hline \multicolumn{2}{|c|}{ Módulo de cámara } \\
\hline Sensor térmico & $\begin{array}{c}\text { Tamaño de pixel de } 17 \mathrm{~mm} \text {, rango } \\
\text { espectral de } 8 \text { a } 14 \mathrm{~mm}\end{array}$ \\
\hline Resolución térmica & $80 \times 60$ \\
\hline Resolución visual & $640 \times 480$ \\
\hline HFOV/VFOV & $46^{\circ} \pm 1^{\circ} / 35^{\circ} \pm 1^{\circ}$ \\
\hline Frecuencia de imagen & $8.7 \mathrm{~Hz}$ \\
\hline Enfoque & Fijo $15 \mathrm{~cm}-$ Infinito \\
\hline Integrado en obturador & Automático/Manual \\
\hline \multicolumn{2}{|c|}{ Radiometría } \\
\hline Rango dinámico de escena & $-20^{\circ} \mathrm{C}-100^{\circ} \mathrm{c}$ \\
\hline Precisión & $\pm 5^{\circ} \mathrm{C} \mathrm{o} 5 \pm \%$ \\
\hline Sensibilidad térmica (MRDT) & $150 \mathrm{mK}$ \\
\hline
\end{tabular}

Fuente: FLIR (2016)

Los parámetros de emisividad y temperatura reflejada fueron medidos por el método de la cinta negra y el método de la reflexión, respectivamente. El primero consiste en la determinación de la emisividad del objeto inspeccionado a través de una cinta negra de emisividad conocida, esta última es colocada en la superficie del objeto. La emisividad se determina a través de varias iteraciones hasta que la temperatura de la estructura y de la cinta negra sea igual. Durante la inspección los valores de la emisividad del concreto variaron de en torno de 0.94 y 0.95 . El método de la reflexión se mide la temperatura de un trozo de aluminio arrugado sobre la superficie de la estructura inspeccionada, asumiendo 1 el valor de emisividad. Es importante mencionar que todos los parámetros fueron calculados para cada termograma obtenido. 
Para el análisis de la diferencia de temperatura entre sectores de la estructura se utilizó la ecuación (1), donde $\Delta T$ es la diferencia de temperatura, $T_{1}$ y $T_{2}$ son las temperaturas de dos sectores diferentes en un mismo elemento.

$$
\Delta T=T_{1}-T_{2}
$$

\section{RESULTADOS}

A continuación, se presentan los resultados obtenidos en la inspección del puente seleccionado. La Figura 2 muestra el detalle de las áreas inspeccionadas. En total son cuatro áreas de la superestructura (cuadros rojos) y cuatro de la infraestructura (cuadros azules).

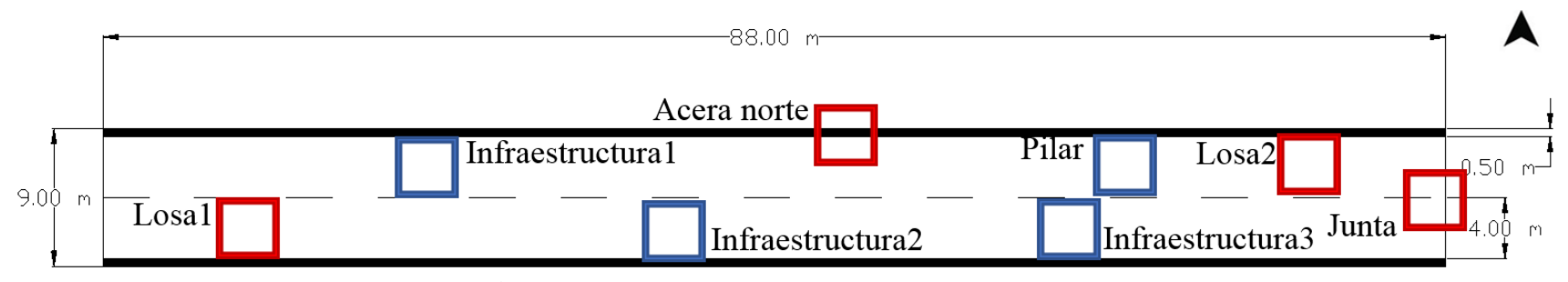

Figura 2. Áreas inspeccionadas con la cámara termográfica

En la Figura 3a se presenta un sector de la losa del puente, en la cual se puede evidenciar la presencia de fisuras; no obstante, adicionalmente en el termograma (Figura 3b) se pueden observar varias áreas calientes las cuales están representadas con el color amarillo. Esta variación de temperatura indica que el hormigón no es homogéneo y presenta defectos internos, una vez que las áreas por encima de los defectos tienden a calentarse más rápido que las áreas sin defectos (Hiasa et al., 2017; Bagavathiappan et al., 2013; Rocha and Póvoas, 2017). La diferencia de temperatura entre estas áreas es de $2.3^{\circ} \mathrm{C}$ ( $\mathrm{Sp} 1$ Sp3) y $2{ }^{\circ} \mathrm{C}(\mathrm{Sp} 2-\mathrm{Sp} 3)$.
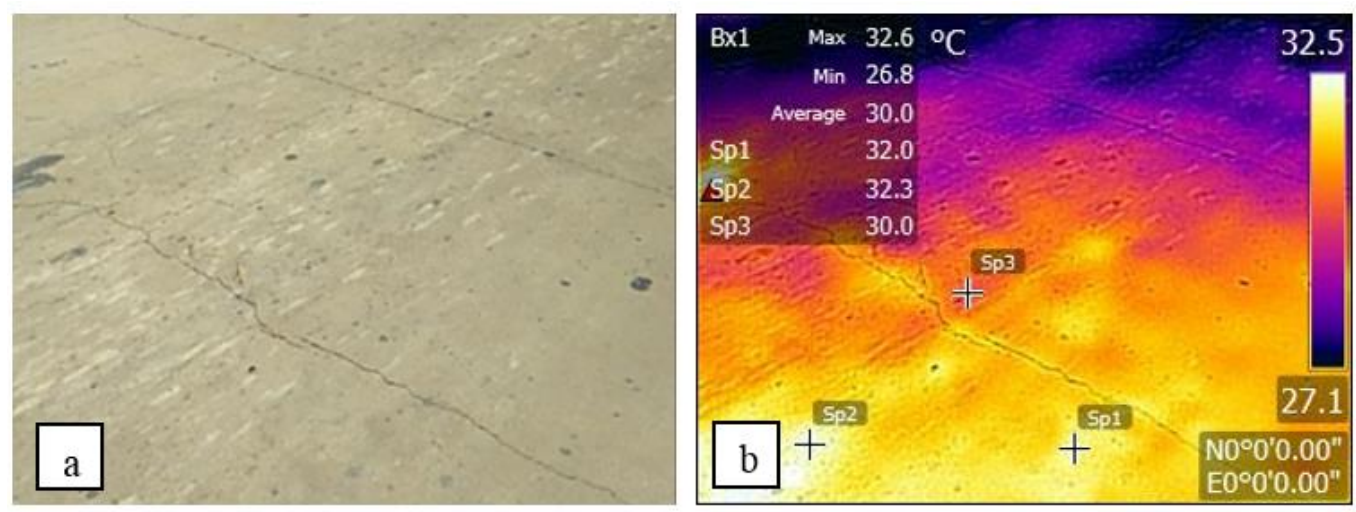

Figura 3. Losa 1: a) Imagen digital y b) termograma

La Figura 4a muestra otro sector de la losa, donde es posible identificar mayor cantidad de fisuras, además de varias áreas calientes (Figura 4b), que ocupan una extensión considerable del termograma, señalando la presencia de áreas destacadas. Los gradientes de temperatura entre las áreas con defectos y el hormigón intacto están por los valores de $2.1^{\circ} \mathrm{C}(\mathrm{Sp} 2-\mathrm{Sp} 4), 2.4^{\circ} \mathrm{C}(\mathrm{Sp} 2-\mathrm{Sp} 1)$ y $2.2^{\circ} \mathrm{C}(\mathrm{Sp} 3-\mathrm{Sp} 1)$. 

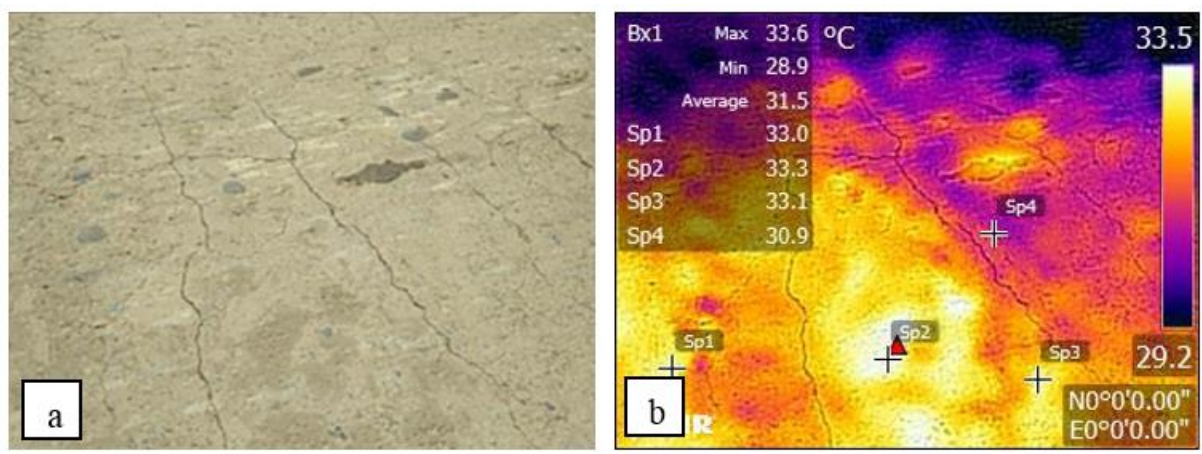

Figura 4. Losa 2: a) Imagen digital y b) termograma

En la Figura 5a se observa la junta del puente en la dirección Este, la cual presenta varios signos de una deterioración avanzada. En el termograma (Figura 5b) se pueden distinguir áreas que presentan mayor temperatura, colores blanco y amarillo, destacándose principalmente en la junta con diferencias de temperatura elevadas, $3.3^{\circ} \mathrm{C}(\mathrm{Sp} 1-\mathrm{Sp} 3)$ y $2.6^{\circ} \mathrm{C}(\mathrm{Sp} 2-\mathrm{Sp} 3)$.
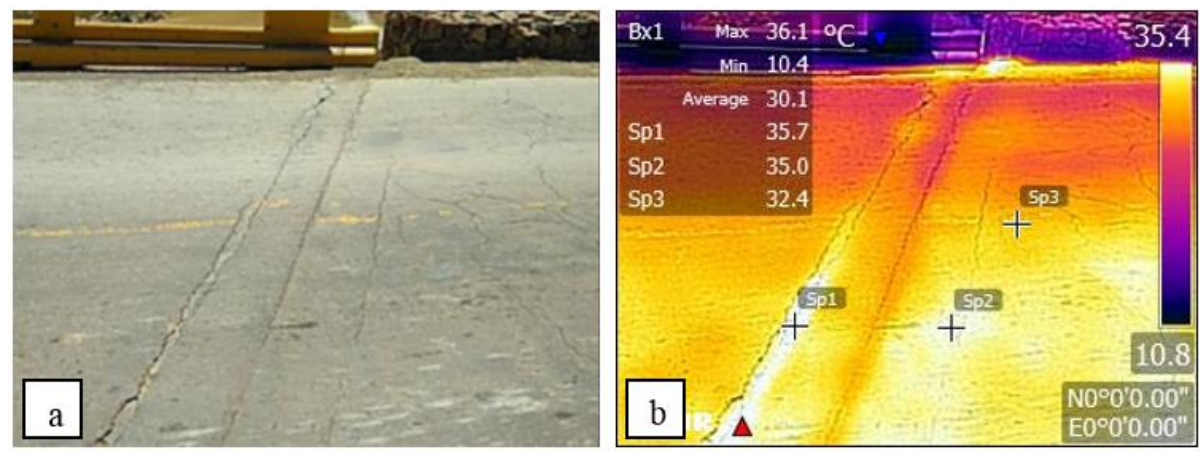

Figura 5. Junta: a) Imagen digital y b) termograma

La acera del puente en dirección Norte se presenta en la Figura 6a, si bien no se observan problemas visibles en la imagen digital, en el termograma (Figura 6b) se pueden apreciar áreas de mayor temperatura, con diferencias de $2.3^{\circ} \mathrm{C}(\mathrm{Sp} 1-\mathrm{Sp} 3)$ y $2.6^{\circ} \mathrm{C}(\mathrm{Sp} 2-\mathrm{Sp} 3)$, que indican anomalías en el hormigón como la falta homogeneidad o, por la magnitud de estas, áreas destacadas.
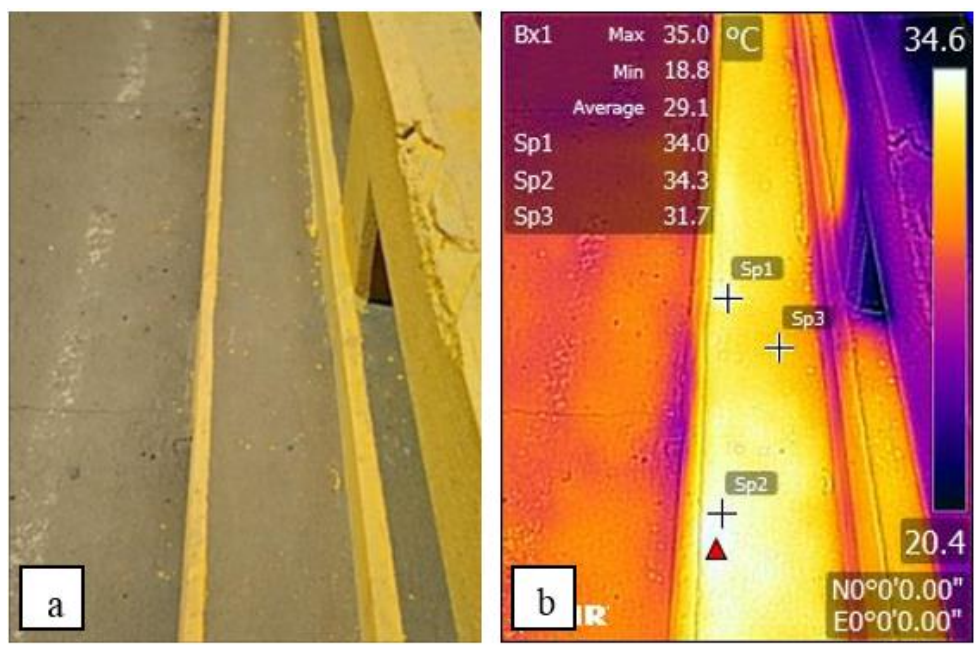

Figura 6. Acera norte: a) Imagen digital y b) termograma 
Con respecto a la infraestructura, la Figura 7a presenta la imagen digital de la losa vista desde la parte inferior, donde no es posible apreciar defectos o problemas visibles. Ya en el termograma (Figura 7b) se detectan algunos sectores calientes; sin embargo, las diferencias de temperatura son menores cuando se comparan con la superestructura, $1.4^{\circ} \mathrm{C}(\mathrm{Sp} 1-\mathrm{Sp} 3)$ y $0.7^{\circ} \mathrm{C}(\mathrm{Sp} 2-\mathrm{Sp} 3)$, esto se debe principalmente a que la infraestructura no recibe directamente la radiación solar y la temperatura ambiente es la única que produce diferencias de temperatura mediante convección.
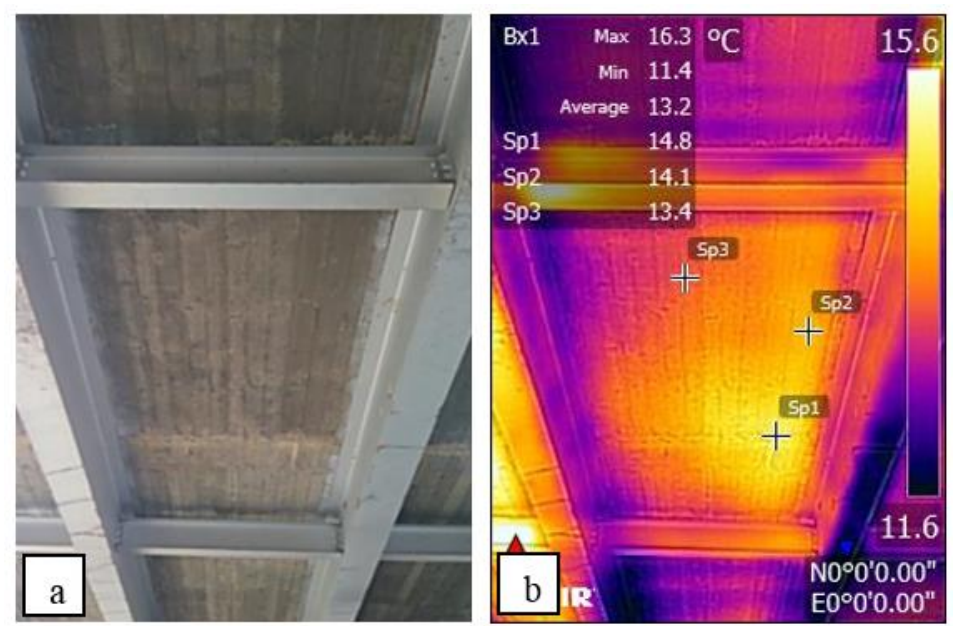

Figura 7. Infraestructura 1: a) Imagen digital y b) termograma

La misma situación puede encontrarse en las Figuras 8 y 9. En el primer caso la diferencia de temperatura es de $1.1^{\circ} \mathrm{C}(\mathrm{Sp} 1-\mathrm{Sp} 3)$ y $0.9^{\circ} \mathrm{C}(\mathrm{Sp} 2-\mathrm{Sp} 3)$. Para la Figura 8 b la diferencia es de $2.2^{\circ} \mathrm{C}(\mathrm{Sp} 1-$ $\mathrm{Sp} 3), 2.1^{\circ} \mathrm{C}(\mathrm{Sp} 2-\mathrm{Sp} 3)$ y $1.1^{\circ} \mathrm{C}(\mathrm{Sp} 4-\mathrm{Sp} 3)$. Es imporante mencionar que la termografia infrarroja detecta irregularidades en el hormigón en ambos casos a pesar de no ser observadas en las imágenes digitales.
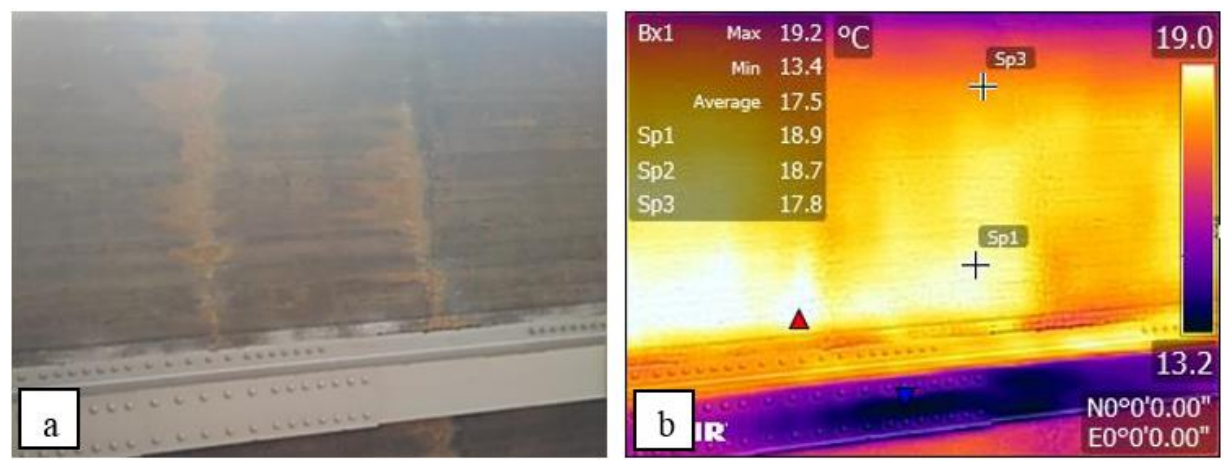

Figura 8. Infraestructura 2: a) Imagen digital y b) termograma
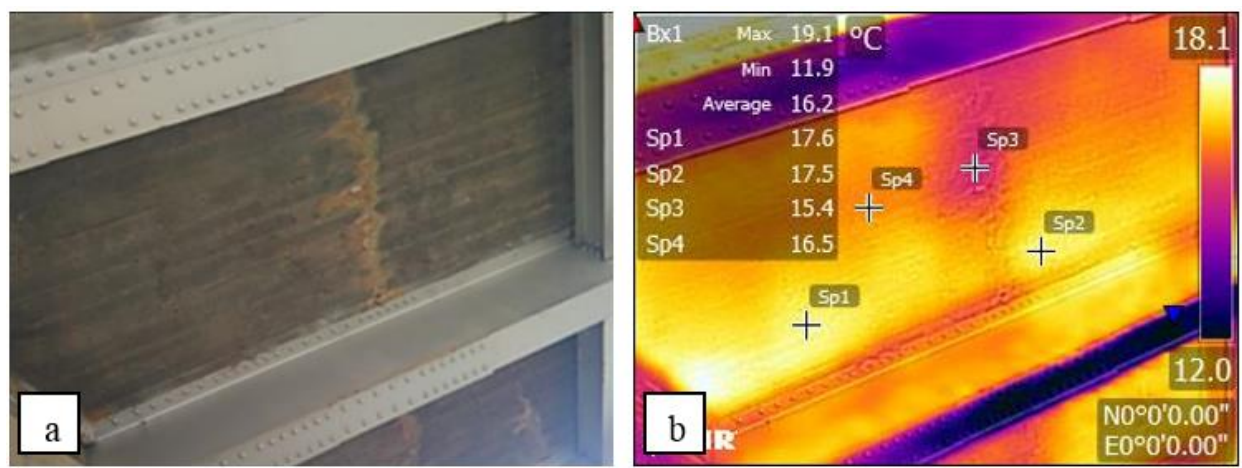

Figura 9. Infraestructura 3: a) Imagen digital y b) termograma 
Finalmente, en la Figura 10b se presenta la imagen térmica de un apoyo intermedio del puente, evidenciando áreas de mayor temperatura, $1^{\circ} \mathrm{C}(\mathrm{Sp} 1-\mathrm{Sp} 2)$, lo que indicaría áreas comprometidas a ser deprendidas o con defectos internos.
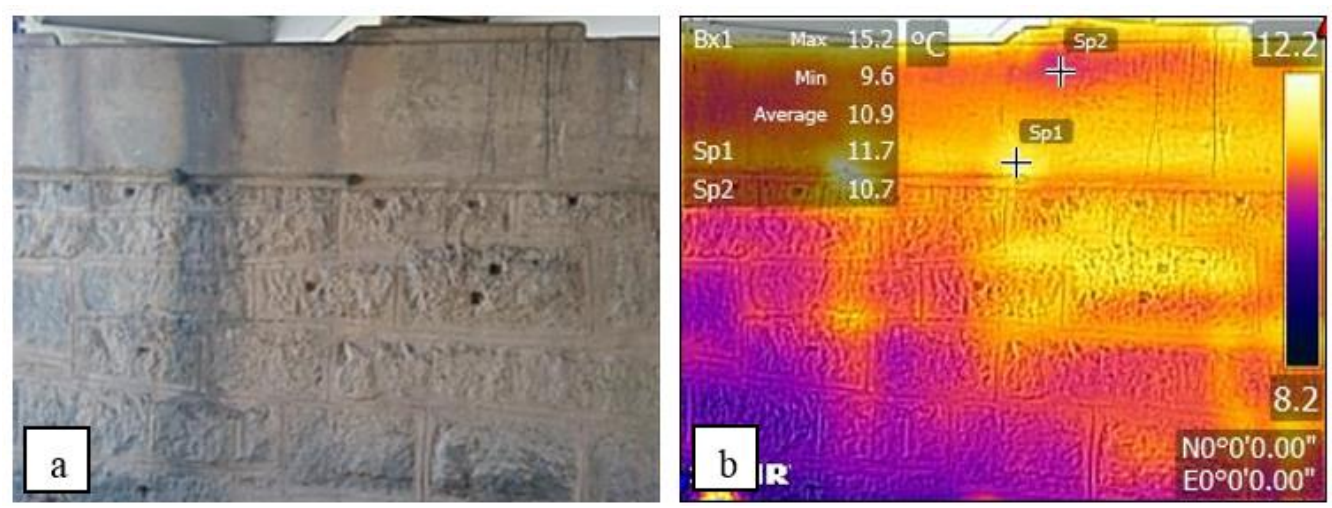

Figura 10. Pilar: a) Imagen digital y b) termograma

De los resultados del presente estudio de caso se comprueba que la termografía infrarroja permite la detección de anomalías en el hormigón, una vez que se pueden detectar áreas de diferentes temperaturas, que normalmente se identifican como defectos internos, delaminaciones o áreas desprendidas (Clark et al., 2003; Hiasa et al., 2016; Rehman et al., 2016; Sultan and Washer, 2017); sin embargo, para caracterizar mejor estos defectos es necesario un monitoreo y la utilización de otros ensayos no destructivos a fin de complementar resultados.

Si bien el ensayo de termografía infrarroja permite la detección de anomalías tanto en la superestructura como la infraestructura, se puede observar que los valores de los gradientes térmicos son diferentes. En el primer sector, las diferencias de temperaturas son elevadas, mayores a $2^{\circ} \mathrm{C}$, lo que facilita su detección con la cámara termográfica; no obstante, en la infraestructura las diferencias son menores, en torno a $1^{\circ} \mathrm{C}$, que si bien permite la detección podría dificultar si las diferencias fueran más reducidas (Clark et at., 2003; Farrag et al., 2016; ASTM, 2013).

\section{CONCLUSIONES}

En el presente artículo fue realizado un estudio de caso con el fin de verificar la aplicabilidad de la termografía infrarroja en la inspección de puentes, específicamente en la localidad de PunataCochabamba, Bolivia.

Se comprobó que la detección de anomalías en el concreto para este tipo de condiciones ambientales es posible; sin embargo, los resultados son mayormente cualitativos, es posible la identificación del área afectada pero no la cuantificación del daño. Además, los resultados varían de acuerdo con el sector inspeccionado, en la superestructura se tiene mayores gradientes térmicos que en la infraestructura.

A pesar de las pocas normas referentes a la inspección de puentes con termografía infrarroja, el ensayo puede ser incorporado en la inspección de estas estructuras como un complemento a la inspección visual $\mathrm{y}$, de esta forma, dar diagnósticos y pronósticos más certeros.

Aunque los puentes sean estructuras importantes, estos no reciben la atención requerida. En Bolivia no existen programas de inspección periódica ni está reglamentado el uso de ensayos como la termografía infrarroja u otros que permitan prevenir e identificar manifestaciones patológicas.

\section{AGRADECIMIENTOS}

A la Universidad Privada del Valle (UNIVALLE). 


\section{REFERENCIAS}

ASTM - American Society for Testing and Materials. (2013b), D4788-03: Standard Test Method for Detecting Delaminations in Bridge Decks Using Infrared Thermography. West Conshohocken, United States. Doi: http://dx.doi.org/10.1520/D4788

Bagavathiappan, S., Lahiri, B., Saravanan, T., Philip, J. (2013), Infrared thermography for condition monitoring - A review. Infrared Physics \& Technology. 60(1):35-55. Doi: https://doi.org/10.1016/j.infrared.2013.03.006

Clark, M., McCann, D., Forde, M. (2003), Application of infrared thermography to the non-destructive testing of concrete and masonry bridges. NDT\&E International. 36(4):265-275. Doi: https://doi.org/10.1016/S0963-8695(02)00060-9

Climate. (2019). Clima Punata. Último acceso: 28 de mayo de 2019. Disponible en: https://es.climatedata.org/america-del-sur/bolivia/cochabamba/punata-51265/

El Deber. (2015). Un estudio planteó reforzar puentes de la vía hace 10 años. Último acceso: 28 de mayo de 2019. Disponible en: https://www.eldeber.com.bo/economia/Un-estudio-planteo-reforzarpuentes-de-la-via-hace-10-anos-20150314-29887.html

Farrag, S., Yehia, S., Qaddoumi, N. (2016), Investigation of Mix-Variation Effect on Defect-Detection Ability Using Infrared Thermography as a Nondestructive Evaluation Technique. Journal of Bridge Engineering. 21(3):1-15. Doi: http://dx.doi.org/10.1061/(ASCE)BE.1943-5592.0000779

FLIR (2016). Smartphone S60 de Cat® Manual del usuario. Último acceso: 28 de mayo de 2019. Disponible en: https://www.catphones.com/download/User-Manuals/S60-Smartphone/S60-Manualdel-usario-Espa\%C3\%B1ol.pdf

Gucunski, N., Imani, A., Romero, F., Nazarian, S., Yuan, D., Wiggenhauser, h., Shokouhi, P., Taffe, A., Kutrubes, D. (2013), "Nondestructive Testing to Identify Concrete Bridge Deck Deterioration". SHRP 2 Research Reports, Transportation Research Board of the National Academies, Washington D.C., United States, p. 85. Doi: https://doi.org/10.17226/22771

Hiasa, S., Birgul, R., Catbas, N. (2016), Infrared thermography for civil structural assessment: demonstrations with laboratory and field studies. Journal of Civil Structural Health Monitoring. 6(3):619-636. Doi: https://doi.org/10.1007/s13349-016-0180-9

Hiasa, S., Birgul, R., Catbas, N. (2017), Investigation of effective utilization of infrared thermography (IRT) through advanced finite element modeling. Construction and Building Materials, 150(30):295309. Doi: https://doi.org/10.1016/j.conbuildmat.2017.05.175

La Opinión. (2015). Caída de puentes en Bolivia. Último acceso: 28 de mayo de 2019. Disponible en: http://www.opinion.com.bo/opinion/articulos/2015/1103/noticias.php?id=174761

Mascia, N., Sartorti, A. (2011), Identificación y análisis de patologías en puentes de carreteras urbanas y rurales. Revista ingeniería de construcción. 26(1):05-24. Doi: https://dx.doi.org/10.4067/S071850732011000100001

Mendes, P., Moreira, M., Pimienta, P. (2012), Pontes de concreto armado: efeitos da corrosão e da variação do módulo de elasticidade do concreto. IBRACON de Estruturas e Materiais. 5(3):389-401. Doi: http://dx.doi.org/10.1590/S1983-41952012000300008

Oh, T., Kee, S., Arndt, R., Popovics, J., Zhu, J. (2013), Comparison of NDT Methods for Assessment of a Concrete Bridge Deck. Journal of Engineering Mechanics. 139(3):305-314. Doi: http://dx.doi.org/10.1061/(asce)em.1943-7889.0000441

Pintan, N., Berenguer, R., Silva, A., Lins, C., Monteiro, E. (2015), Pathological Manifestations and the Study of Corrosion Present on Bridges of the City of Recife. Electronic Journal of Geotechnical Engineering. 20(24):11893-11907.

Rehman, S., Ibrahim, Z., Memon, S. A., Jameel, M. (2016), Nondestructive test methods for concrete bridges: A review. Construction and Building Materials. 107(15):58-86. Doi: https://doi.org/10.1016/j.conbuildmat.2015.12.011 
Rocha, J., Póvoas, Y. (2017), Infrared thermography as a non-destructive test for the inspection of reinforced concrete bridges: A review of the state of the art. Revista ALCONPAT, 7(3):200-214. Doi: https://dx.doi.org/10.21041/ra.v7i3.223

Rocha, J., Póvoas, Y. (2019), Detection of Delaminations in Reinforced Concrete Bridges Using Infrared Thermography. Revista ingeniería de construcción. 34(1):55-64. Doi: https://dx.doi.org/10.4067/S0718-50732019000100055

Sultan, A., Washer, G. (2017), A pixel-by-pixel reliability analysis of infrared thermography (IRT) for the detection of subsurface delamination. NDT \& $E$ International, 92:177-186. Doi: https://doi.org/10.1016/i.ndteint.2017.08.009

Vaghefi, K., Ahlborn, T., Harris, D., Brooks, C. (2015), Combined Imaging Technologies for Concrete Bridge Deck Condition Assessment. Journal of Performance of Constructed Facilities. 29(4):1-8. Doi: http://dx.doi.org/10.1061/(ASCE)CF.1943-5509.0000465

Watase, A., Birgul, R., Hiasa, S., Matsumoto, M., Mitani, K., Catbas, F. (2015), Practical identification of favorable time windows for infrared thermography for concrete bridge evaluation. Construction and Building Materials. 101(1):1016-1030. Doi: https://doi.org/10.1016/j.conbuildmat.2015.10.156 